\title{
The Mass of the Graviton and the Cosmological Constant Puzzle
}

\section{Mario Novello*}

Instituto de Cosmologia Relatividade e Astrofísica (ICRA-BR/CBPF),

Rua Dr. Xavier Sigaud, 150, CEP 22290-180

Rio de Janeiro, Brazil

E-mail: novello@cbpf.br

We propose an interpretation of the cosmological constant puzzle - i.e., the enormous value of the ratio $\rho_{P l} / \rho_{v a c} \approx 10^{120}$ - in terms of the total number of gravitons in the observable universe based on a recently discovered relationship between $\Lambda$ and the mass $m_{g}$ of the graviton.

Fifth International Conference on Mathematical Methods in Physics - IC2006

April 24-28 2006

Centro Brasileiro de Pesquisas Fisicas, Rio de Janeiro, Brazil

${ }^{*}$ Speaker. 


\section{Introduction}

It has often been argued that there is no relation between the cosmological constant and a possible non-null mass of the graviton. Such a claim is based on comparing the equation of evolution of weak metric perturbations satisfying Einstein's first gravitational theory with the equation of a spin-2 massless field propagating in the Minkowski background. However, it has recently been shown [1] that this approach relies heavily on the interpretation of the cosmological constant as a special configuration states of matter. This analysis uses Einstein's first description of his gravitational field theory, hereafter refered to as $E I$, given by the equations of motion

$$
R_{\mu v}-\frac{1}{2} R g_{\mu v}=-\kappa T_{\mu v}
$$

In this framework the cosmological constant is nothing but the energy content of the matter in a special configuration state called vacuum in which the corresponding stress-energy tensor takes the form $T_{\mu \nu}=\Lambda g_{\mu \nu}$. Here $\Lambda$ should not be understood as a fundamental quantity of its own, but instead is related to the - classical or quantum - matter content of the universe. Our purpose here is not to deal with such an effective $\Lambda$, namely a complex quantity depending on the presence of matter vacuum. Instead we are concerned with the bare fundamental cosmological constant. In the case in which $\Lambda$ is treated as a fundamental new constant related to the gravitational interaction, one should adopt Einstein's second description of the equations of motion, hereafter refered to as EII:

$$
R_{\mu v}-\frac{1}{2} R g_{\mu v}+\Lambda g_{\mu v}=-\kappa T_{\mu v}
$$

Then proving that $\Lambda$ and a non-zero $m_{g}$ are not correlated by perturbing the Minkowski geometry does not make sense, since in the absence of matter $g_{\mu \nu}=\eta_{\mu \nu}$ is not even a solution of equation (1.2). Thus the standard argument claiming there is no relation between $\Lambda$ and $m_{g}$ cannot be used [2].

In order to analyze the consequences of a similar argument in the realm of the theory given by eq. (1.2) one needs to make some important modifications. The first one is to look for the equivalent fundamental state of the geometry in this theory, namely the one containing the maximum number of symmetries generated by the ten Killing vectors. This means dealing with the deSitter geometry. Thus, one needs to examine the equations of motion of spin- 2 massive and massless fields in such geometry, and then compare these equations with the evolution of a small perturbation $\delta g_{\mu \nu}$ of the deSitter geometry under the theory EII. This is done in reference [1]. The relevant result is that the equation for the perturbation $\delta g_{\mu \nu}$ of the deSitter background is identical to a spin-2 massive field, the mass of which is proportional to $\sqrt{\Lambda}$. This property remains valid not only for deSitter but also for arbitrary background geometries [3] as long as we stay in the setting of theory EII.

\section{Two masses?}

What does one expect the mass associated to $\Lambda$ to be? Following the standard procedure in the quantum context, the natural quantity should be constructed using three basic ingredients: $\Lambda$, Planck's constant $\hbar$ and the light velocity $c$. We are thus led, up to a numerical factor which, for the 
time being, we take of the order of the unity, to the formula

$$
m_{g}=\frac{\hbar \sqrt{\Lambda}}{c} .
$$

Using relativity in the quantum world, this is the only way to obtain a formula for mass using a quantity which has the dimension of length. Neverthless, as it has often been emphasized, the graviton is not just one of those particles that happens to exist in any metrical structure: it is special. This is because the graviton is intrinsically related to the metrical structure of space-time. However, one can construct another quantity which has the dimension of mass using $\Lambda$. It also contains three basic ingredients, but exhibits a dependency on the gravity world instead of the quantum world. This second mass, call it $M_{g}$, is constructed using $\Lambda$, Newton's constant $G_{N}$ and the light velocity $c$. This yields, up to a numerical factor which, for the time being, we take of the order of the unity, to the expression

$$
M_{g}=\frac{c^{2}}{G_{N} \sqrt{\Lambda}} .
$$

Before proceeding let us stop for a while to ponder about the meaning of this $M_{g}$. This can be rewritten in an equivalent form as

$$
M_{g}=\frac{\Lambda c^{4}}{G_{N}} \frac{1}{\sqrt{\Lambda^{3}}} \frac{1}{c^{2}}
$$

This formula contains three separate terms. The first one represents the energy density generated by $\Lambda$; the second one is the total volume of the universe restricted to its horizon $\left(c / H_{o}\right)^{2} \approx \Lambda^{-1}$ and the last term converts the total energy into a mass. Thus we are almost constrained to interpret $M_{g}$ as the total mass of all existing gravitons in the observable universe. Writing

$$
M_{g}=N_{g} m_{g}
$$

it then follows that $N_{g}$ is nothing but the total number of gravitons contained inside the observable horizon.

An unexpected result appears when we evaluate this quantity in our actual universe: it is exactly the same number that appears in the standard cosmological constant puzzle. Indeed, from the above expressions we obtain:

$$
N_{g}=\frac{c^{3}}{\hbar G_{N} \Lambda}
$$

and for the ratio of the Planck density and the vacuum density one has

$$
\frac{\rho_{P l}}{\rho_{v a c}} \approx \frac{c^{7}}{\hbar G_{N}^{2}} \frac{G_{N}}{c^{4} \Lambda} .
$$

Consequently,

$$
\frac{\rho_{P l}}{\rho_{v a c}} \approx N_{g} \approx 10^{120}
$$

This analysis suggests an explanation to the traditional cosmological constant problem: the value of $\rho_{P l} / \rho_{v a c}$ is so large because there is a huge quantity $N_{g}$ of massive gravitons in the observable universe, , with $m_{g} \approx \sqrt{\Lambda}$. 


\section{Acknowledgements}

This work was partially supported by Conselho Nacional de Desenvolvimento Científico e Tecnológico (CNPq) and Fundação de Amparo à Pesquisa do Estado do Rio de Janeiro (FAPERJ) of Brazil. I would like to thank Dr Samuel Senti for his kind help in the final English version of this manuscript.

\section{References}

[1] M. Novello and R. P. Neves, Class. Quantum Grav. 20 (2003) L67-L73. See also M. Novello and R. P. Neves, Class. Quantum Grav. 19, 5335-5351 (2002).

[2] Let us note that in another traditional argument against a massive graviton there is a misinterpretation of the generalization of the Yukawa analysis of field theory to the deSitter background. It has been stated (see for instance the definition for the limit imposed on $m_{g}$ used by the Particle Data Group in Review of Particle Physics published by the European Physical Journal) that, if the graviton had a mass, then the gravitational interaction would not be long-range. This concerns the Yukawa potential that, in Minkovski geomety, is given by

$$
\Phi(r)=\frac{\exp (-\mu r)}{r} .
$$

However, the analysis of the compatibility of a massive graviton and gravitational effects should be stated in the deSitter background instead of the Minkowski background. In the zero curvature case, a massive field theory has a radius of influence which depends on the absolute value of the mass. In deSitter background a functional of the curvature (that is $\Lambda$ ) already provides a typical length, thus the Yukawa analysis cannot be extended to the deSitter geometry. A direct calculation shows that this is indeed the case. We will come back to this in a separate paper with R.P. Neves and S. E. P. Bergliaffa.

[3] A comment on this identification is worthwhile. In the Minkowski geometry the Poincare group of the symmetries of space-time has two Casimir operators that yields two label for the associated irreducible unitary representation (IUR), identified as the mass and the spin. In the case of the deSitter group $S O(4,1)$ there are also two Casimir operators but they cannot be identified to the spin and the mass in all cases. For simplicity and tradition's sake, we still refer to the deSitter parameters as mass and spin, even when the contraction IUR of the deSitter group is not a IUR of Poincare group.

Let us also point out that although the mass of the graviton given in equation (2.1) is proportional to $\sqrt{\Lambda}$, the corresponding gravitational perturbation has only two degrees of freedom (see [1] for details). 